\title{
Deficit hídrico e secamento parcial do sistema radicular em pomar de lima ácida
}

\author{
Antonio Hélder Rodrigues Sampaio(1), Maurício Antonio Coelho Filho(2), Eugênio Ferreira Coelho (2), \\ Rossini Daniel (1), Victor Vinícius Machado( ${ }^{(1)}$, Gian Carlos Carvalho( ${ }^{(1)}$ e Edvaldo Bispo Santana Junior(1) \\ (1)Universidade Federal do Recôncavo da Bahia, s/no, CEP 44380-000 Cruz das Almas, BA, E-mail: helderagronomo@hotmail.com, \\ rossini.pi@gmail.com, gian_carvalho@hotmail.com, viniciusufrb@yahoo.com.br, edvaldobispo@gmail.com ${ }^{(2)}$ Embrapa Mandioca e Fruticultura \\ Tropical, Caixa Postal 007, CEP 44380-000 Cruz das Almas, BA. E-mail: macoelho@cnpmf.embrapa.br, ecoelho@cnpmf.embrapa.br
}

Resumo - O objetivo deste trabalho foi avaliar o efeito do deficit hídrico associado ao secamento parcial do sistema radicular ("partial rootzone drying", PRD) na produção e eficiência de uso de água, em pomar de lima ácida 'Tahiti', na Região do Semiárido baiano. Utilizou-se o delineamento de blocos ao acaso, com seis tratamentos e seis repetições. Os tratamentos com PRD foram realizados pela alternância periódica $(7,14$ ou 21 dias) dos lados em que o microaspersor localizava-se em relação às plantas. Esses tratamentos foram conduzidos com lâmina d'água equivalente a $50 \%$ da evapotranspiração da cultura (ETc) e comparados ao tratamento com deficit hídrico de $50 \%$ da ETc com microaspersor fixo em um dos lados das plantas, e ao tratamento $100 \%$ da ETc, com aspersores de ambos os lados das plantas. Avaliou-se também o PRD (21 dias) com diminuição da irrigação em $25 \%$ da ETc. O PRD com 50\% da ETc não difere do tratamento com esse mesmo deficit e sem alternância de lados da irrigação, em termos de produtividade, eficiência do uso da água, e diâmetro e peso dos frutos, independentemente do período de alternância dos lados de irrigação. O PRD com diminuição da irrigação em $25 \%$ da ETc não causa perdas em relação à condição sem deficit e sem localização da irrigação.

Termos para indexação: Citrus latifolia, citros, eficiência de uso de água, limão Tahiti.

\section{Water deficit and partial rootzone drying in an acid lime orchard}

\begin{abstract}
The objective of this work was to evaluate the effect of water deficit and of partial rootzone drying (PRD) on the yield and water use efficiency in a 'Tahiti' lime orchard, in the Semiarid Region of Bahia state, Brazil. The experiment was carried out in a randomized block design, with six treatments and six replicates. Treatments with PRD were carried out by the periodic alternance $(7,14$ or 21 days) of the sides in which the micro sprinklers were located in relation to the plants. These treatments were performed with a water depth equivalent to $50 \%$ of crop evapotranspiration (ETc). They were compared to the treatment with $50 \%$ water deficit, using one fixed micro sprinkler at one of the plant sides, and to the treatment at $100 \%$, with sprinklers at both plant sides. PRD (21 days) with $25 \%$ ETc water depth reduction was also evaluated. PRD at 50\% ETc do not differ from the treatment with this same water deficit and without alternating irrigation sides for productivity, water use efficiency, and fruit diameter and weight, regardless of the irrigation alternating period. PRD with 25\% ETc water depth reduction do not cause losses in comparison to the condition without deficit and without localized irrigation.
\end{abstract}

Index terms: Citrus latifolia, citrus, water use efficiency, Tahiti lemon.

\section{Introdução}

A agricultura irrigada é responsável pelo uso de 69\% da água consumida no mundo, e a tendência de escassez dos recursos hídricos, no contexto do aquecimento global, torna necessário o aumento da eficiência do uso da água pelas culturas (EUA) e a otimização dos sistemas de irrigação. Uma das alternativas para aumentar a EUA é a redução da transpiração ou evapotranspiração, para as quais algumas metodologias são utilizadas, como a do deficit hídrico regulado em fases de crescimento vegetativo (RDI) das culturas, e a do próprio uso de sistemas de irrigação localizada.

A partir da década de 90, simultaneamente ao uso do RDI, estudos para otimização da irrigação com emprego de deficit hídrico foram desenvolvidos por meio do uso da técnica de secamento parcial do sistema radicular (PRD, "partial rootzone drying"). Esta técnica é constituída da alternância do lado de irrigação no sistema radicular: enquanto parte das raízes é irrigada, a outra parte é exposta ao secamento do solo. Os lados de umedecimento e secamento são alternados com

Pesq. agropec. bras., Brasília, v.45, n.10, p.1141-1148, out. 2010 
uma frequência que depende do tipo de solo e da planta analisada (Kriedemann \& Goodwin, 2003).

O deficit hídrico pela estratégia de PRD tem mostrado que é possível aumentar a eficiência de uso da água pelas culturas, com redução do crescimento vegetativo, mantendo-se a produção em um patamar satisfatório (Dry \& Loveys, 1998). A teoria defendida é a de que o sistema radicular passa a produzir uma quantidade maior de ácido abscísico (ABA), que se transloca pelo eixo vascular, concentra-se na parte aérea e ocasiona o fechamento parcial dos estômatos e, consequentemente, resulta em menor perda de água para a atmosfera (Gowing et al., 1990; Davies \& Zhang, 1991; Davies et al., 2002).

Uma pequena redução na abertura estomática pode reduzir substancialmente a perda de água, com um mínimo de efeito sobre a absorção de $\mathrm{CO}_{2}$ e sobre o processo fotossintético (Jones, 1992). Com a diminuição da utilização de água no processo de transpiração, que é responsável por mais de $95 \%$ da água absorvida pela planta (Comstock, 2002) e pela manutenção de produtividade e qualidade do produto, haverá aumento da eficiência de uso da àgua pelas plantas.

As pesquisas realizadas nos últimos anos com PRD vêm tentando responder a questão sobre como e com que intensidade o mecanismo bioquímico desencadeado por esta técnica influencia a produtividade de uma cultura. Há relatos sobre a economia de água alcançada pelo método (Kang et al., 1998), bem como sobre o maior desenvolvimento do sistema radicular (Melgar et al., 2010) e controle na abertura de estômatos (Raveh, 2008). Contudo, observa-se que na maioria das culturas a aplicação do deficit hídrico convencional alcança resultados de produtividade semelhantes, a exemplo dos trabalhos de Pudney \& McCarthy (2004) com uva, Kirda et al. (2007) com tangerina, Treeby et al. (2007) e Dzikiti et al. (2008) com a laranja-de-umbigo.

Os estudos com PDR em citros ainda são incipientes, e não há um consenso dos resultados da técnica, em razão da heterogeneidade dos solos cultivados e da variação de respostas de crescimento da planta nas diferentes estações do ano (Melgar et al., 2010), sobretudo em regiões subtropicais onde as chuvas se concentram no verão. No Brasil, são poucos os estudos que utilizam a técnica de PRD, a exemplo do de Silva (2005) para a cultura da videira.

O objetivo deste trabalho foi avaliar o efeito do deficit hídrico associado ao secamento parcial do sistema radicular na produção e eficiência de uso de água, em um pomar de lima ácida 'Tahiti' no Semiárido baiano.

\section{Material e Métodos}

O estudo foi conduzido na fazenda Boa Vista, pertencente à Iaçu Agropastoril Ltda., no Município de Iaçu, no Semiárido baiano $\left(12^{\circ} 46^{\prime} \mathrm{S}\right.$ e $40^{\circ} 13^{\prime} \mathrm{W}$, a $280 \mathrm{~m}$ de altitude), de maio de 2008 a agosto de 2009 , em pomar de lima ácida 'Tahiti' (Citrus latifolia Tanaka) com cinco anos de idade, enxertada em limão 'Cravo' (Citrus limonia Osbeck), em espaçamento de 8x5 m. Utilizou-se sistema de irrigação por microaspersão, com emissores de vazão de 19 L por hora.

Utilizou-se o delineamento experimental de blocos ao acaso, com seis tratamentos e seis repetições. Cada parcela experimental foi composta por uma planta que, em conformidade com os tratamentos aplicados, era irrigada com um ou dois microaspersores localizados a $2,5 \mathrm{~m}$ do tronco. Os tratamentos de PRD foram promovidos pela alternância do uso de um microaspersor adicional no lado oposto à linha de aspersores, em intervalos de 7, 14 e 21 dias (T1, T2 e T3, respectivamente), com aplicação de $50 \%$ da evapotranspiração da cultura (ETc). Esses tratamentos foram comparados ao da irrigação com deficit hídrico convencional $(50 \% \mathrm{ETc})$, em que se utilizou um microaspersor fixo, que molhava apenas um lado do sistema radicular da planta (T4), e ao tratamento testemunha (T5), que recebeu o dobro do volume de água (100\% ETc), aplicado por dois microaspersores, um em cada lado da planta. Também foram comparados ao tratamento T6 (PRD), com redução de $25 \%$ da lâmina total aplicada, tendo-se alternado o uso de 1 e 2 microaspersores por planta a cada 21 dias.

$\mathrm{O}$ regime de secamento e umedecimento do solo foi monitorado em cada lado da planta, por meio da técnica de reflectometria de domínio no tempo, com sondas de $10 \mathrm{~cm}$ de comprimento, calibradas para o solo, conforme Coelho et al. (2006), localizadas a $30 \mathrm{~cm}$ de profundidade e posicionadas a 1,0 e $1,5 \mathrm{~m}$ do caule. O sistema radicular foi avaliado em março de 2009, a partir de estudos de densidade de raiz, obtida com amostragem nos dois lados da planta, nas distâncias de 1,0 e $1,5 \mathrm{~m}$ do caule, nas profundidades de $0-0,20$, $0,20-0,40$ e $0,40-0,60 \mathrm{~m}$. 
A resistência estomática e o potencial de água na folha foram avaliados por meio de porômetro de marca Delta-T, modelo AP4 (Delta-T Devices, Cambridge, Inglaterra), e bomba de pressão tipo 'Scholander' modelo 3115 (Soilmoisture Equipment Corp., Santa Barbara, Estados Unidos), respectivamente. Esses parâmetros fisiológicos foram avaliados em um bloco selecionado aleatoriamente na fase final do experimento. As leituras ocorreram em quatro quadrantes - norte, sul, leste e oeste -, entre 11 e 14 horas. A resistência estomática foi medida em folhas fisiologicamente maduras, entre a primeira e a quinta folha após o ápice do ramo, a partir de 1,5 $\mathrm{m}$ de altura do solo. O potencial de água na folha foi determinado em ramos constituídos de 4 a 6 folhas, com diâmetro médio de 10 a $20 \mathrm{~mm}$, retirados sempre em posição exposta ao sol, entre 1,5 e 1,8 $\mathrm{m}$ do solo, conforme Cotrim (2008).

O volume de água aplicado ao tratamento testemunha foi estimado pela evapotranspiração da cultura para irrigação localizada (ETc), calculado por meio de medidas de evapotranspiração de referência (ETo), coeficiente de cultura $(\mathrm{Kc})$ e fator de localização (Kr) pela equação (Keller \& Bliesner, 1990): $\mathrm{ETc}=$ ETo $\mathrm{x} \mathrm{Kc} \times \mathrm{Kr}$, em que $\mathrm{Kr}$ é dado por: 0,10 $\mathrm{PAS}^{0,5}$, e PAS é a percentagem de área molhada, definida como a relação entre área molhada pelo microaspersor e a área ocupada pela planta.

A precipitação pluvial e a evapotranspiração de referência (ETo) foram registradas, durante todo experimento, por pluviômetro e tanque classe A, respectivamente, localizados a $800 \mathrm{~m}$ do local do experimento (Tabela 1). A adubação foi realizada por meio de fertirrigações distribuídas durante os meses de realização do experimento, com $113 \mathrm{~kg} \mathrm{ha}^{-1}$ de nitrogênio, $52 \mathrm{~kg} \mathrm{ha}^{-1}$ de fósforo e $113 \mathrm{~kg} \mathrm{ha}^{-1}$ de potássio. Para os tratamentos em PRD, em razão da redução do volume de água aplicado, os fertilizantes foram adicionados manualmente, em quantidade necessária para equiparar a adubação do tratamento controle, na mesma frequência das fertirrigações.

O período de colheita ocorreu de agosto de 2008, época que os tratamentos tinham 80 dias de aplicação, a julho de 2009. A eficiência do uso de água (EUA) foi calculada em todos os tratamentos, tendo-se considerado a produtividade total dos frutos e a lâmina bruta de água aplicada pela irrigação, conforme a equação $\mathrm{EUA}=\mathrm{P} / \mathrm{LBA}$, em que: $\mathrm{P}$ é a produtividade em $\mathrm{kg} \mathrm{ha}^{-1}$, e LBA é a lâmina bruta de água aplicada no pomar, em metros cúbicos por hectare.

$\mathrm{O}$ peso médio e o diâmetro equatorial de frutos também foram analisados. Procedeu-se à coleta de dez frutos por planta, que foram acondicionados em sacos de plásticos previamente identificados e levados ao laboratório de fisiologia vegetal, da Embrapa Mandioca e Fruticultura Tropical, onde foram pesados em balança analítica. Posteriormente, mediu-se o diâmetro equatorial de frutos, com o auxílio de um paquímetro universal (Multimedição Comércio de Equipamentos Ltda., São Paulo, Brasil).

As análises estatísticas foram realizadas pelo programa Sisvar, por meio de análise de variância e teste de Tukey para as variáveis densidade de raiz e potencial hídrico dos ramos. Para os parâmetros de produção de frutos, EUA, diâmetro e peso médio de frutos, utilizou-se o teste de Scott-Knott, a 5\% de probabilidade. Também foram correlacionados os parâmetros de resistência estomática e potencial de água na folha.

\section{Resultados e Discussão}

O secamento e o umedecimento do solo, nos tratamentos T1, T2 e T3, submetidos ao manejo com secamento parcial do sistema radicular, diferenciaram-se dos demais tratamentos por apresentar variação da umidade do solo, em cada lado da planta, que oscilou entre a capacidade de campo e ponto de murcha permanente, em consequência da alternância de lados

Tabela 1. Precipitação pluvial mensal acumulada, evapotranspiração de referência (ETo) e da cultura, para irrigação localizada (ETc), e coeficiente de cultura (Kc) utilizado no pomar de citrus, de junho de 2008 a julho de 2009.

\begin{tabular}{|c|c|c|c|c|c|c|c|c|c|c|c|c|c|c|}
\hline \multirow[t]{2}{*}{ Variável } & \multicolumn{7}{|c|}{2008} & \multicolumn{7}{|c|}{2009} \\
\hline & Jun. & Jul. & Ago. & Set. & Out. & Nov. & Dez. & Jan. & Fev. & Mar. & Abr. & Maio & Jun. & Jul. \\
\hline Precipitação (mm) & 43,0 & 21,5 & 11,0 & 10,0 & 7,0 & 143,5 & 125,0 & 119,5 & 73,0 & 11,0 & 103,0 & 42,0 & 8,0 & 49,0 \\
\hline ETo (mm) & 111,0 & 120,0 & 153,0 & 166,0 & 200,0 & 216,0 & 159,0 & 155,0 & 144,0 & 229,0 & 134,0 & 124,0 & 110,0 & 120,0 \\
\hline $\mathrm{ETc}(\mathrm{mm})$ & 52,3 & 57,4 & 73,1 & 81,8 & 94,7 & 105,7 & 77,9 & 74,7 & 70,1 & 108,9 & 60,6 & 61,7 & 54,5 & 56,6 \\
\hline Kc adotado & 1,01 & 0,83 & 0,73 & 0,76 & 0,84 & 0,77 & 0,60 & 0,73 & 0,66 & 0,73 & 0,73 & 0,86 & 1,10 & 1,09 \\
\hline
\end{tabular}


da aplicação de água pelo microaspersor (Figura $1 \mathrm{~A}$, $\mathrm{B}$ e C). No tratamento de deficit hídrico fixo a $50 \%$ ETc (T4), e na testemunha com 100\% ETc (T5), a umidade do solo foi constante ao longo do tempo, de acordo com suas características de aplicação de água (Figura $1 \mathrm{D}$ e E).

O manejo de irrigação com deficit hídrico e o secamento parcial do sistema radicular, com redução da lâmina tanto em 25 como em $50 \%$ da ETc, não apresentaram efeito sobre a densidade de raízes, nos pontos amostrados, em comparação à testemunha
(Tabela 2). No tratamento T3, foi observada maior densidade de raízes a $1,0 \mathrm{~m}$ do caule na camada $0-0,20 \mathrm{~m}$, em relação à camada de $0,4-0,6 \mathrm{~m}$ de profundidade. A densidade de raízes apresentou variabilidade muito alta, com coeficientes de variação superiores a $100 \%$. Verificou-se, também, que a porção de solo não irrigada do tratamento T4 teve densidade radicular compatível com os demais tratamentos, o que contribuiu para a absorção de água e nutrientes no período úmido do ano.

Apesar de que não houve comprovação estatística que diferenciasse os tratamentos, houve a tendência de
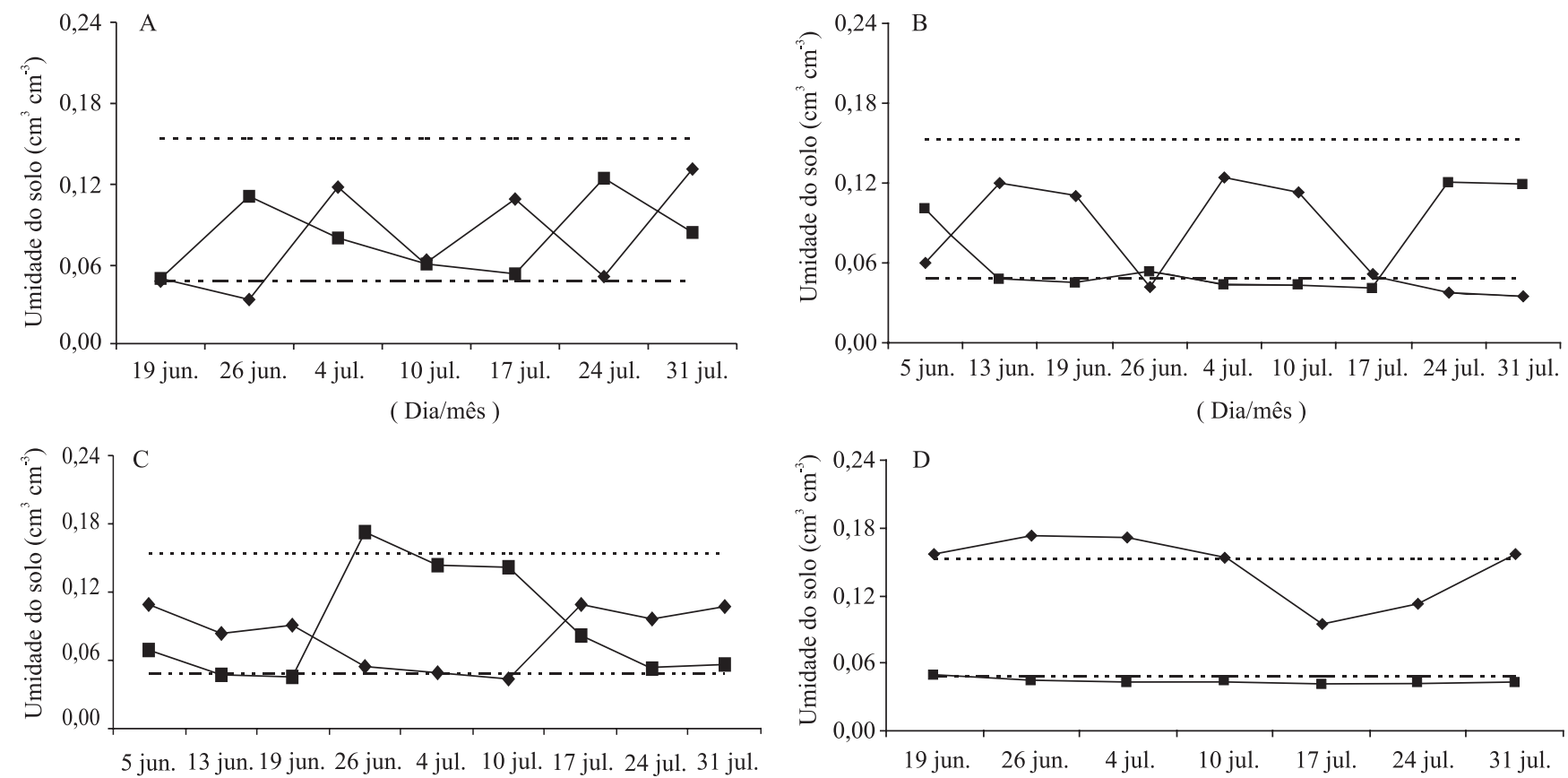

( Dia/mês )
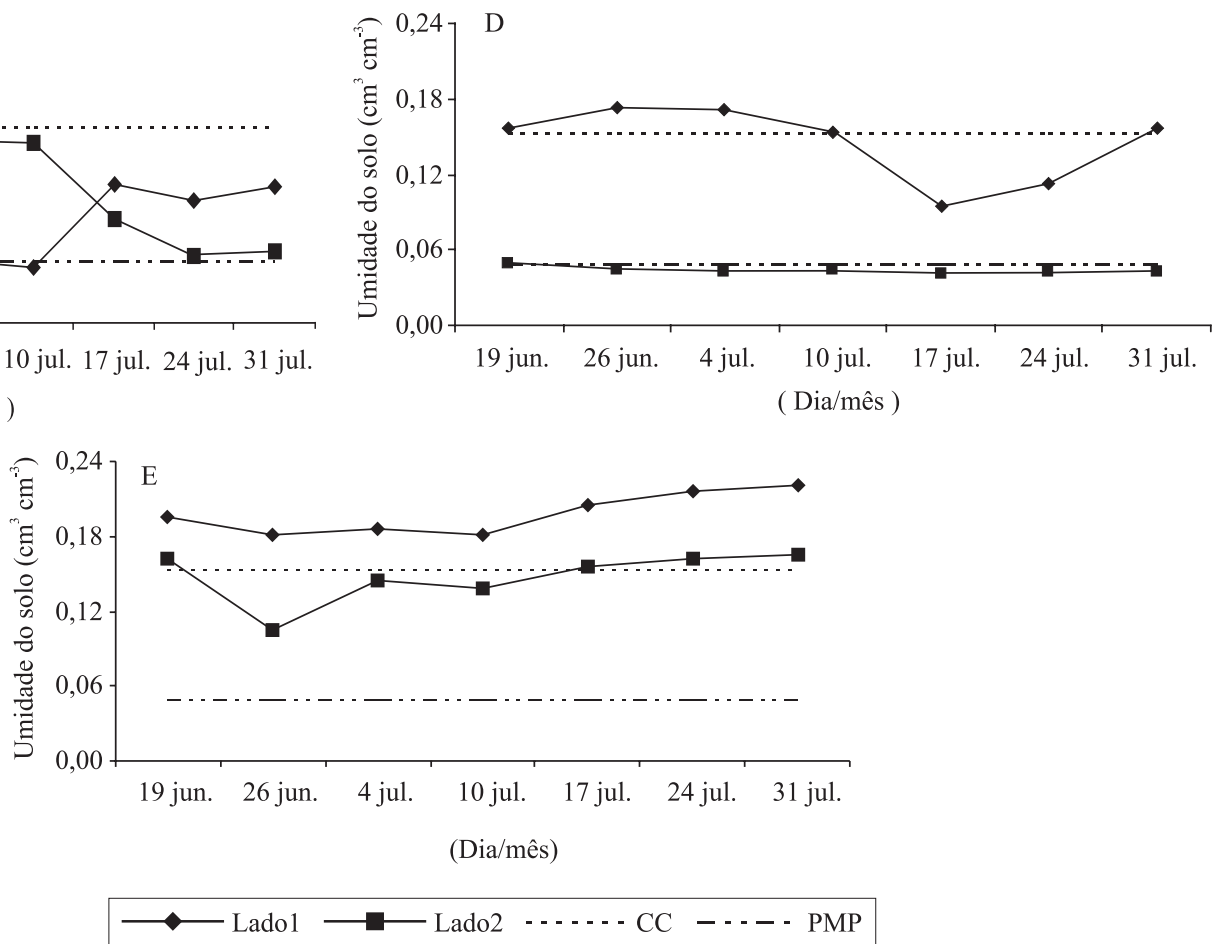

Figura 1. Umidade do solo a $0,30 \mathrm{~m}$ de profundidade, em cada lado de plantas de lima ácida 'Tahiti', irrigadas por microaspersor e submetidas aos tratamentos: T1 (A), PRD 7 dias; T2 (B), PRD 14 dias; T3 (C), PRD 21 dias; T4 (D), deficit fixo; e T5 (E), testemunha; CC, capacidade de campo; PMP, ponto de murcha permanente. 
ocorrerem maiores valores de densidades de raiz nos tratamentos T3, T5 e T6, principalmente nas camadas de 0-0,20 m, (Tabela 2). A tendência de maior densidade radicular em T3 pode estar relacionada à indução do crescimento de raízes secundárias, em resposta ao maior secamento do solo, o que estaria de acordo com a teoria de Stoll et al. (2000). Em condições controladas, houve comprovação do efeito do PRD sobre o aumento da densidade radicular, nos trabalhos de Melgar (2010) com citros e de Guang-Cheng (2008) com pimentas. Uma justificativa abordada por Spollen et al. (2000) é a de que o manejo de irrigação, com secamento do sistema radicular, favorece o crescimento de raízes pela inibição da produção de etileno pelo ABA.

O potencial hídrico dos ramos das plantas em T5 $(-1,21 \mathrm{MPa})$ foi significativamente superior $(\mathrm{p}<0,05)$ ao dos demais tratamentos, seguido de T6 (-1,57 MPa) e T2 (-1,77 MPa). Os tratamentos T1, T3 e T4 tiveram os menores potenciais hídricos, com $-2,18,-2,02$ e $-2,1 \mathrm{MPa}$, respectivamente. De acordo com Medina et al. (2005), na citricultura, potenciais hídricos foliares iguais ou menores que -2,0 $\mathrm{MPa}$ são considerados severos. Em estudo de resposta ao deficit hídrico em mudas de limão 'Cravo' e citrumelo 'Swingle', combinados com cinco variedades de copa, foram constatados valores de potencial hídrico foliar igual -2,08 MPa, após 16 dias de deficit hídrico induzido, enquanto as plantas irrigadas tiveram média de $-0,48 \mathrm{MPa}$ (Donato et al., 2007).

O potencial hídrico dos ramos teve forte correlação com a resistência estomática. De acordo com o modelo de regressão, o aumento da resistência estomática está relacionado à redução do potencial hídrico (Figura 2). A correlação inversa entre resistência estomática e potencial hídrico foliar também foi verificada em trabalhos de Nogueira et al. (2000), em pitanga cultivada em casa de vegetação, e de Silva et al. (2003), em três espécies lenhosas nativas da Caatinga.

Os tratamentos de PRD tiveram resistência estomática entre 7,3 a $21,8 \mathrm{~cm} \mathrm{~s}^{-1}$, para a faixa de potencial entre -1,7 a -2,25 MPa, enquanto a testemunha (T5) teve resistência de 3,9 a $7,3 \mathrm{~cm} \mathrm{~s}^{-1}$ e potencial hídrico dos ramos entre $-1,1$ a $-1,7 \mathrm{MPa}$. Estudos em plantas adultas de limão (Citrus limon), na região de Negev, Israel, mostraram valores de resistência estomática de 9,9, 14,6 e 31,7 $\mathrm{cm} \mathrm{s}^{-1}$, em tratamentos com suprimento da necessidade hídrica ideal, tratamento de PRD com redução de $50 \%$ da lâmina total aplicada e suspensão da irrigação durante 60 dias, respectivamente (Raveh, 2008). De acordo com Syvertsen (1982), o potencial hídrico mínimo que coincide com o fechamento estomático varia de $-1,6$ a $-3,5 \mathrm{MPa}$, a depender do estádio de maturação da folha, e tem relação direta com a taxa de renovação de folhas, que é um importante mecanismo de ajuste das plantas ao estresse hídrico.

A produção de frutos nos tratamentos $\mathrm{T} 5$ e T6 foi superior à dos demais com produtividade (Tabela 3), tendo-se equiparado à média nacional, que é $22,43 \mathrm{Mg} \mathrm{ha}^{-1}$ para pomares não irrigados com cinco anos de idade (Food and Agriculture Organization of the United Nations, 2009). Este resultado mostra que o PRD com redução de $25 \%$ do volume de água aplicada não teve efeito negativo sobre a produtividade. Em relação aos tratamentos com $50 \%$ de redução da

Tabela 2. Densidade de raiz $\left(\mathrm{cm} \mathrm{cm}^{-3}\right)$ em diferentes camadas de solo e distâncias das plantas de lima ácida 'Tahiti', submetidas a tratamentos de deficit hídrico e secamento parcial do sistema radicular, em março de $2009^{(1)}$.

\begin{tabular}{|c|c|c|c|c|c|c|c|c|}
\hline Camada (m) & T1 & $\mathrm{T} 2$ & T3 & T4i & T4ni & T5 & T6 & CV $(\%)$ \\
\hline & \multicolumn{8}{|c|}{ Densidade de raiz a $1,0 \mathrm{~m}$ do caule } \\
\hline $0,0-0,2$ & $0,17 \mathrm{aA}$ & $0,18 \mathrm{aA}$ & $0,62 \mathrm{aA}$ & $0,31 \mathrm{aA}$ & $0,21 \mathrm{aA}$ & $0,46 \mathrm{aA}$ & $0,42 \mathrm{aA}$ & 101,06 \\
\hline $0,2-0,4$ & $0,11 \mathrm{aA}$ & $0,05 \mathrm{aA}$ & $0,13 \mathrm{aA}$ & $0,07 \mathrm{aA}$ & $0,20 \mathrm{aA}$ & $0,30 \mathrm{aA}$ & $0,23 \mathrm{aA}$ & 115,59 \\
\hline $0,4-0,6$ & $0,04 \mathrm{aA}$ & $0,11 \mathrm{aA}$ & $0,09 \mathrm{aC}$ & $0,08 \mathrm{aA}$ & $0,10 \mathrm{aA}$ & $0,13 \mathrm{aA}$ & $0,17 \mathrm{aA}$ & 118,84 \\
\hline \multirow[t]{2}{*}{ CV (\%) } & 105,42 & 164,74 & 138,22 & 166,75 & 73,65 & 67 & 107,88 & \\
\hline & \multicolumn{8}{|c|}{ Densidade de raiz a $1,5 \mathrm{~m}$ do caule } \\
\hline $0,0-0,2$ & $0,12 \mathrm{aA}$ & $0,27 \mathrm{aA}$ & $0,50 \mathrm{aA}$ & $0,44 \mathrm{aA}$ & $0,17 \mathrm{aA}$ & $0,61 \mathrm{aA}$ & $0,68 \mathrm{aA}$ & 143,19 \\
\hline $0,2-0,4$ & $0,18 \mathrm{aA}$ & $0,36 \mathrm{aA}$ & $0,29 \mathrm{aA}$ & $0,16 \mathrm{aA}$ & $0,31 \mathrm{aA}$ & $0,30 \mathrm{aA}$ & $0,22 \mathrm{aA}$ & 93,98 \\
\hline $0,4-0,6$ & $0,17 \mathrm{aA}$ & $0,16 \mathrm{aA}$ & $0,14 \mathrm{aA}$ & $0,14 \mathrm{aA}$ & $0,37 \mathrm{aA}$ & $0,31 \mathrm{aA}$ & $0,20 \mathrm{aA}$ & 133,96 \\
\hline CV (\%) & 94,07 & 84,88 & 151,37 & 94,05 & 79,50 & 95,00 & 152,82 & \\
\hline
\end{tabular}

(1)Médias seguidas por letras iguais, minúsculas nas linhas e maiúsculas nas colunas, não diferem entre si pelo teste de Tukey, a 5\% de probabilidade. T1, PRD 7 dias; T2, PRD 14 dias; T3, PRD 21 dias; T4i, deficit hídrico fixo, lado irrigado; T4ni, lado que não recebeu irrigação; T5, testemunha; e T6, deficit hídrico a $25 \%$ da ETc. 
lâmina, foi constatado um decréscimo na produção entre 24,8 a $44,6 \%$. Não foram comprovadas vantagens da alternância dos lados da irrigação sobre o tratamento com deficit fixo (T4). Considerando-se os valores brutos de produtividade, observou-se entre os tratamentos de PRD um desempenho melhor, apesar de não significativo, para o tratamento com inversão dos lados a cada 7 dias (T1).

Kirda et al. (2007), em estudos realizados com PRD na Turquia, em tangerina (Citrus reticulata cultivar Marisol), mostraram a redução de $10 \%$ na produção de frutos no tratamentos de PRD com redução de 50\%

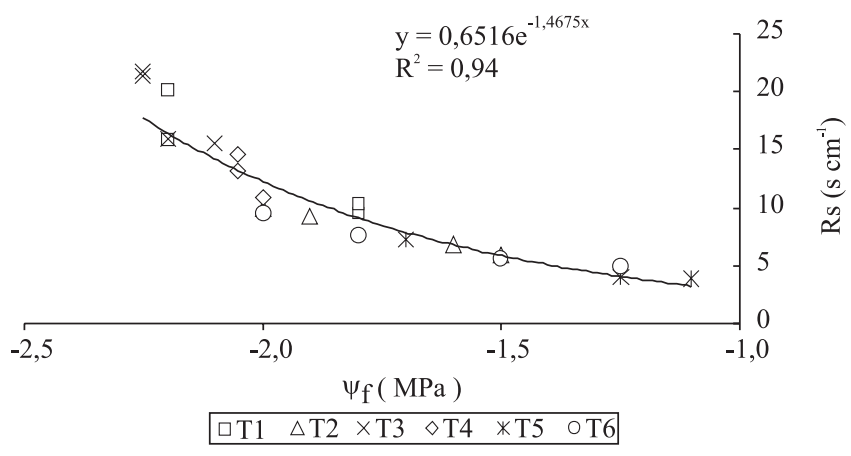

Figura 2. Relação entre potencial hídrico nos ramos e resistência estomática (Rs) da lima ácida 'Tahiti' em condição de sol, submetidas aos tratamentos: T1, PRD 7 dias; T2, PRD 14 dias; T3, PRD 21 dias; T4, deficit fixo; T5, tratamento testemunha; e T6, deficit hídrico a $25 \%$ da ETc, em 28 de agosto de 2009.

Tabela 3. Produtividade de frutos, eficiência de uso de água (EUA) e diâmetro e peso médio de frutos de lima ácida 'Tahiti', submetida à irrigação e a secamento parcial do sistema radicular, de agosto de 2008 a julho de $2009^{(1)}$.

\begin{tabular}{lcccr}
\hline Tratamento & $\begin{array}{c}\text { Produção anual } \\
\left(\mathrm{Mg} \mathrm{ha}^{-1}\right)\end{array}$ & $\begin{array}{c}\text { EUA } \\
\left(\mathrm{kg} \mathrm{m}^{-3}\right)\end{array}$ & $\begin{array}{c}\text { Diâmetro de } \\
\text { frutos }(\mathrm{cm})\end{array}$ & $\begin{array}{r}\text { Peso de } \\
\text { frutos }(\mathrm{g})\end{array}$ \\
\hline T1 & $17,12 \mathrm{~b}$ & $9,32 \mathrm{a}$ & $5,37 \mathrm{a}$ & $94,92 \mathrm{a}$ \\
T2 & $12,82 \mathrm{~b}$ & $6,98 \mathrm{a}$ & $5,43 \mathrm{a}$ & $96,73 \mathrm{a}$ \\
T3 & $15,93 \mathrm{~b}$ & $8,67 \mathrm{a}$ & $5,53 \mathrm{a}$ & $101,71 \mathrm{a}$ \\
T4 & $17,41 \mathrm{~b}$ & $9,48 \mathrm{a}$ & $5,24 \mathrm{a}$ & $88,21 \mathrm{a}$ \\
T5 & $23,16 \mathrm{a}$ & $6,30 \mathrm{a}$ & $5,49 \mathrm{a}$ & $97,80 \mathrm{a}$ \\
T6 & $22,49 \mathrm{a}$ & $8,16 \mathrm{a}$ & $5,47 \mathrm{a}$ & $98,00 \mathrm{a}$ \\
\hline Média & 18,16 & 8,15 & 5,43 & 96,65 \\
CV $(\%)$ & 29,45 & 27,39 & 2,73 & 7,19 \\
\hline
\end{tabular}

(1)Médias seguidas por letras iguais pertencem ao mesmo grupo pelo teste de Scott-Knott, a 5\% de probabilidade. T1, PRD 7 dias; T2, PRD 14 dias; T3, PRD 21 dias; T4, deficit hídrico fixo; T5, testemunha; e T6, deficit hídrico a $25 \%$ da ETc. da lâmina de irrigação, em relação à testemunha sem deficit. Resultados com laranja-de-umbigo (Citrus sinensis), na região semiárida da África do Sul, com precipitação de 700 a $800 \mathrm{~mm}$, a redução da lâmina de irrigação em 50\% não afetou a produtividade, e a alternância de irrigação não teve efeito em relação ao tratamento com esse deficit hídrico e irrigação fixa a um dos lados da planta (Dzikiti et al., 2008).

Em relação às variáveis diâmetro e peso médio de fruto, não houve diferença significativa entre os tratamentos (Tabela 3). Isto mostra a rusticidade dos citrus, mais especificamente do limoeiro, a condições de restrição de água. Em trabalhos desenvolvidos por Alves Júnior (2006), em pomar de lima ácida 'Tahiti' com quatro anos de idade, não foi observada diferença na produtividade de frutos, diâmetro de frutos $\mathrm{e}$ espessura da casca, quanto aos níveis de irrigação de $25,50,75$ e $100 \%$ da evapotranspiração de referência. No entanto, estudos com a técnica de PRD, com uso de deficit de 40 a $50 \%$ da ETc, constataram reduções do diâmetro e peso médio de frutos em tangerina (Kirda et al., 2007) e laranja-de-umbigo (Treeby et al., 2007). Contudo, nesses estudos, o tratamento com deficit hídrico com irrigação fixa a um dos lados das plantas teve resultados semelhantes aos tratamentos de deficit com PRD.

Os tratamentos com deficit hídrico não apresentaram diferenças quanto à eficiência de uso de água (Tabela 3 ). Ao se observarem os valores brutos de EUA, nota-se que houve aumento não significativo nos resultados dos tratamentos com deficit hídrico, em comparação à testemunha (T5). Kirda et al. (2007) obsevaram, em dois anos de estudos com tangerina, a variação da eficiência de uso de água entre 4 e $5,6 \mathrm{~kg} \mathrm{~m}^{-3}$, para o tratamento com $100 \%$ da ETc, e de 9 a $15 \mathrm{~kg} \mathrm{~m}^{-3}$, em tratamentos com deficit de $50 \%$ da ETc.

Sadras (2009) realizou estudo de meta-análise em um grupo de dados relacionados à eficiência do uso da água, quanto à técnica do PRD, deficit hídrico e irrigação convencional, para diversas culturas, e constatou que, em $80 \%$ dos casos, a EUA foi maior com a utilização do deficit hídrico, PRD ou deficit hídrico com irrigação fixa a um dos lados, com acréscimos que variaram em torno de $20 \%$. Entretanto, apenas em $20 \%$ dos casos, a EUA para tratamentos submetidos ao PRD foi superior ao deficit hídrico com irrigação fixa a um dos lados das plantas. 


\section{Conclusões}

1. A técnica do secamento parcial do sistema radicular associada ao deficit hídrico de $50 \%$ da evapotranspiração da cultura não difere do tratamento com deficit e sem alternância de lados da irrigação, em termos de produtividade, eficiência do uso da água, e diâmetro e peso dos frutos, independentemente do período de alternância dos lados de irrigação.

2. A técnica do secamento parcial do sistema radicular associada à diminuição da irrigação em $25 \%$ da evapotranspiração da cultura não causa perdas em relação à condição sem deficit e sem localização da irrigação.

\section{Referências}

ALVES JÚNIOR, J. Necessidade hídrica e resposta da cultura de lima ácida 'Tahiti' a diferentes níveis de irrigação. 2006. 100p. Tese (Doutorado) - Escola Superior de Agricultura Luiz de Queiroz, Piracicaba.

COELHO, E.F.; VELlAME, L. de M.; COELHO FILHO, M.A.; LEDO, C.A. da S. Desempenho de modelos de calibração de guias de onda acopladas a TDR e a multiplexadores em três tipos de solos. Revista Brasileira de Ciência do Solo, v.30, p.23-30, 2006.

COMSTOCK, J.P. Hydraulic and chemical signalling in the control of stomatal conductance and transpiration. Journal of Experimental Botany, v.53, p.195-200, 2002.

COTRIM, C.E. Otimização da irrigação localizada em pomares de manga no semi-árido baiano. 2008. 177p. Tese (Doutorado) Universidade Federal de Viçosa, Viçosa.

DAVIES, W.J.; WILKINSON, S.; LOVEYS, B. Stomatal control by chemical signalling and the exploitation of this mechanism to increase water use efficiency in agriculture. New Phytologist, v.153, p.449-460, 2002.

DAVIES, W.J.; ZHANG, J.H. Root signals and the regulation of growth and development of plants in drying soil. Annual Review of Plant Physiology and Plant Molecular Biology, v.42, p.55-70, 1991.

DONATO, S.L.R.; PEREIRA, C.S.; BARROS, Z. de J.; SIQUEIRA, D.L. de.; SALOMÃO, L.C.C. Respostas de combinações de variedades copa e porta-enxerto de citros à deficiência hídrica. Pesquisa Agropecuária Brasileira, v.42, p.1507-1510, 2007.

DRY, P.R.; LOVEYS, B.R. Factors influencing grapevine vigour and the potential for control with partial rootzone drying. Australian Journal of Grape and Wine Research, v.4, p.140-148, 1998.

DZIKITI, S.; STEPPE, K.; LEMEUR, R. Partial rootzone drying of drip irrigated 'Navel' orange trees [Citrus sinensis (L.) Osbeck] under semi-arid tropical conditions. Acta Horticulturae, n.792, p.249-256, 2008.

FOOD AND AGRICULTURE ORGANIZATION OF THE UNITED NATIONS. FAO statistical yearbook. 2009. Disponível em: <http://www.faostat.fao.org/siti/339/default.aspx>. Acesso em: 29 mar. 2010.

GOWING, D.J.; DAVIES, W.J.; JONES, H.G. A positive root-sourced signal as an indicator of soil drying in apple, Malus $\mathrm{x}$ domestica Borkh. Journal of Experimental Botany, v.41, p.1535-1540, 1990.

GUANG-CHENG, S.; ZHAN-YU, Z.; NA, L.; SHUANG-EN, Y.; WENG-GANG, X. Comparative effects of deficit irrigation (DI) and partial rootzone drying (PRD) on soil water distribution, water use, growth and yield in greenhouse grown hot pepper. Scientia Horticulturae, v.119, p.11-16, 2008.

JONES, H.G. Plants and microclimate: a quantitative approach to environmental plant physiology. $2^{\text {nd }}$ ed. Cambridge: Cambridge University, 1992. 428p.

KANG, S.; LIANG, Z.; HU, W.; ZHANG, J. Water use efficiency of controlled alternate irrigation on root-divided maize plant. Agricultural Water Management, v.38, p.69-76, 1998.

KELLER, J.; BLIESNER, R.D. Sprinkle and trickle irrigation. New York: Avibook, 1990. 649p.

KIRDA, C.; TOPALOGLU, F.; TOPÇU, S.; KAMAN, H. Mandarin yield response to partial root drying and conventional deficit irrigation. Turkish Journal of Agriculture and Forestry, v.31, p.1-10, 2007.

KRIEDEMANN, P.E.; GOODWIN, I. Regulated deficit irrigation and partial rootzone drying. Canberra: Land \& Water Australia, 2003. 102p. (Irrigation insights, 4).

MEDINA, C.L.; MACHADO, E.C.; RENA, A.B.; SIQUEIRA, D.L. de. Fisiologia dos citros. In: MATTOS JÚNIOR, D.; DE NEGRI, J.D.; PIO, R.M.; POMPEU JÚNIOR, J. (Ed.). Citros. Campinas: IAC, 2005. p.147-195.

MELGAR, J.C.; DUNLOP, J.M.; SYVERTSEN, J.P. Growth and physiological responses of the citrus rootstock Swingle citrumelo seedlings to partial rootzone drying and deficit irrigation. Journal of Agricultural Science, v.148, p.593-602, 2010.

NOGUEIRA, R.J.M.C.; SILVA JUNIOR, J.F.; BEZERRA, E.F.; LEDERMAN, I.E.; BURITY, H.A.; SANTOS, V.F. Comportamiento estomático y tensión de água em el xilema de dos genótipos de pianga (Eugenia uniflora L.) cultivados bajo estrés hídrico. Investigacion Agrária: Production y Proteccion Vegetal, v.15, p.49-61, 2000.

PUDNEY, S.; MCCARTHY, M.G. Water use efficiency of field grown Chardonnay grapevines subjected to partial rootzone drying and deficit irrigation. Acta Horticulturae, n.664, p.567-573, 2004.

RAVEH, E. Partial root-zone drying as a possible replacement for 'Verdelli' practice in lemon production. Acta Horticulturae, n.792, p.537-541, 2008.

SADRAS, V.O. Does partial root-zone drying improve irrigation water productivity in the field? A meta-analysis. Irrigation Science, v.27, p.183-190, 2009.

SILVA, E.C. da.; NOGUEIRA, R.J.M.C.; AZEVEDO NETO, A.D. de.; SANTOS, V.F. dos. Comportamento estomático e potencial 
da água da folha em três espécies lenhosas cultivadas sob estresse

hídrico. Acta Botânica Brasileira, v.17, p.231-246, 2003.

SILVA, J.A.M. Irrigação lateralmente alternada e com deficit controlado na videira cv. Petite Syrah. 2005. 92p. Dissertação (Mestrado) - Universidade Federal de Viçosa, Viçosa.

SPOLLEN, W.G.; LENOBLE, M.E.; SAMUELS, T.D.; BERNSTEIN, N.; SHARP, R.E. Abscisic acid accumulation maintains maize primary root elongation at low water potentials by restricting ethylene production. Plant Physiology, v.122, p.967-976, 2000.
STOLL, M.; LOVEYS, B.; DRY, P. Whole plant integration and agriculture exploitation. Hormonal changes induced by partial rootzone drying of irrigated grapevine. Journal of Experimental Botany, v.51, p.1627-1634, 2000.

SYVERTSEN, J.P. Minimum leaf water potential and stomatal closure in citrus leaves of different ages. Annals of Botany, v.49, p.827-834, 1982.

TREEBY, M.T.; HENRIOD, R.E.; BEVINGTON, K.B.; MILNE, D.J.; STOREY, R. Irrigation management and rootstock effects on navel orange [Citrus sinensis (L.) Osbeck] fruit quality. Agricultural Water Management, v.91, p.24-32, 2007.

Recebido em 30 de junho de 2010 e aprovado em 29 de outubro de 2010 